\title{
Sequential Enrichment of Sulfated Glycans by Strong Anion-Exchange Chromatography Prior to Mass Spectrometric Measurements
}

\author{
Ming Lei, Milos V. Novotny, and Yehia Mechref \\ National Center of Glycomics and Glycoproteomics, Department of Chemistry, Indiana University, \\ Bloomington, Indiana, USA
}

\begin{abstract}
Structural characterization of sulfated glycans through mass spectrometry (MS) has been often limited by their low abundance in biological materials and inefficient ionization in the positive-ion mode. Here, we describe a microscale method for sequentially enriching sulfated glycans according to their degree of sulfation. This method is based on modifying the binding ability of strong anion-exchange material through the use of different sodium acetate concentrations, thus enabling fairly selective binding and a subsequent elution of different glycans according to their degree of sulfation. Before this enrichment, the negative charge on the sialic acid, which is commonly associated with such glycans, was eliminated through permethylation that is used to enhance the positive-ion mode matrix-assisted laser desorption/ionization time-of-flight mass spectrometry (MALDI-MS) signal for all glycans. This enrichment approach minimizes competitive ionization between sulfated and neutral glycans, as well as that between sulfated species with a different degree of sulfation. The described method was initially optimized using sulfated oligosaccharide standards, while its potential has been verified for the sulfated N-glycans originated from the bovine thyroid-stimulating hormone (bTSH), a glycoprotein possessing mono- and disulfated N-glycans. This enhancement of the MALDI-MS signal facilitates analysis of some otherwise undetected components. (J Am Soc Mass Spectrom 2010, 21, 348-357) ( 2010 Published by Elsevier Inc. on behalf of American Society for Mass Spectrometry
\end{abstract}

S ulfation of glycans is involved in many biological events, including a fast clearance of luteinizing hormone and thyroid-stimulating hormone [1-3], and lymphocyte homing [4-6]. Sulfated glycans are also found on the envelope of human immunodeficiency virus (HIV) [7], where they may play an important role in the transportation process. More recently, sulfated glycans have also been linked to neural system dysfunctions [8,9] and cancer [10-13]. Consequently, improved analytical characterization of sulfated glycans appears desirable to facilitate the understanding of their biological roles and help elucidating the disease mechanisms at the molecular level. This knowledge could ultimately lead to the discovery of clinically useful biomarkers and, perhaps more importantly, new drug targets [11, 14].

In previous studies, sulfated glycans were characterized through radioactive isotope labeling $[2,15,16]$ and nuclear magnetic resonance (NMR) spectrometry [17, 18]. These approaches either involve hazardous reagents or suffer from inadequate sensitivity. More recently, mass spectrometry (MS) has emerged as a rapid,

Address reprint requests to Dr. Y. Mechref and M. V. Novotny, Department of Chemistry, Indiana University, 800 E Kirkwood Ave, Bloomington, IN 47405, USA. E-mail: ymechref@indiana.edu and novotny@indiana.edu sensitive, and high throughput technology that has now been widely employed in various studies involving glycans [19-21]. However, structural determination and quantitative measurements of sulfated glycans through MS is inherently challenging due to several reasons. First, the presence of a sulfate group suppresses ionization in the positive-ion MS [19-21], which cannot be easily replaced by the negative-ion mode for the reasons of sensitivity as well as the capability to analyze the sulfated and neutral species at the same time. Second, the signal due to glycans with a high degree of sulfation can be suppressed by those with a low degree of sulfation, even if the sulfated glycans were isolated from the neutral oligosaccharides. Third, the presence of other acidic glycoconjugates, such as sialylated glycans, will also interfere with the MS analysis of sulfated glycans. Although numerous investigators have pursued the ionization enhancement of sulfated glycans, such as the use of tripeptide (KKK) ion-pairing method [22-24], only limited effort has been thus far extended to the development of separation protocols enhancing the structural characterization of sulfated glycans through MALDI-MS.

Chromatographic methods capable of isolating sulfated glycans have been explored in the past, including weak anion-exchange (WAX) [10, 25-27], as well as the combination of WAX and other chromatographic tech-
(C) 2010 Published by Elsevier Inc. on behalf of American Society for Mass Spectrometry. $1044-0305 / 10 / \$ 32.00$

doi:10.1016/j.jasms.2009.09.017
Published online September 30, 2009

Received July 16, 2009

Revised September 16, 2009

Accepted September 21, 2009 
niques [28]. However, WAX matrixes are not ideal separation media for sulfated glycans for their inability to resolve sulfated and sialylated molecules without additional treatments [28]. On the other hand, by combining WAX with other separational techniques (e.g., reversed-phase chromatography [26] and capillary electrophoresis [29]), sulfated glycans can be fractionated and resolved according to the properties primarily recognized by the second dimension. However, multidimensional separations are time-consuming and may suffer from some sample losses.

Here, we demonstrate an alternative approach that is capable of isolating sulfated glycans according to their degree of sulfation after permethylation treatment, which eliminates the negative charges associated with sialylated structures. Besides this charge elimination on the sialylated glycans, ionization in the positive-ion mode has been enhanced through derivatization [30, 31]. Next, the permethylated glycans are sequentially subjected to a series of silica-based strong anion-exchange (SAX) spin-columns, which were preconditioned with 750, 500, and $200 \mathrm{mM}$ sodium acetate aqueous solution ( $\mathrm{NaOAc}$ ) and $0.1 \mathrm{M}$ hydrochloric acid aqueous solution $(\mathrm{HCl})$. NaOAc was chosen because $\mathrm{OAc}^{-}$exhibits a relatively weak eluting strength and the buffer would not interfere with MS analysis [32]. Glycans with a decreasing degree of sulfation are retained on the spin-columns, while the neutral molecules can be recovered from the pass-through and washing solutions. The retained sulfated glycans are then eluted with $1 \mathrm{M} \mathrm{NaCl}$ in $5 \%$ acetonitrile $(\mathrm{ACN})$ aqueous solution and desalted through a $\mathrm{C}_{18}$ hydrophobic column. All eluted fractions are subsequently analyzed by matrix-assisted laser desorption/ionization-mass spectrometry (MALDI-MS). The overall procedure requires a minimum amount of glycoprotein sample ( $\mu \mathrm{g}$ scale) and can be completed in $20 \mathrm{~min}$. As the spin-columns are individually optimized for each sulfation state, this system can be used to selectively enrich structures with a given sulfation state. We applied this method to both sulfated oligosaccharide standards as well as the Nglycans isolated from bovine thyroid-stimulating hormone (bTSH), which is a well-studied glycoprotein expressed in bovine anterior pituitary $[2,33,34]$.

\section{Experimental}

\section{Materials}

Mono-, di-, tri-, and tetrasulfated oligosaccharide standards were purchased from V-labs (Covington, LA, USA). Bovine thyroid-stimulating hormone (bTSH), peptide: $\mathrm{N}$-glycosidase $\mathrm{F}$ (PNGase F), pancreatic bovine ribonuclease $B$, fetuin from fetal calf serum, human $\alpha_{1}$-acid glycoprotein, sodium dodecylsulfate (SDS), octyl phenoxylpolyethoxylethanol, a nonionic and nondenaturing detergent (NP-40), and the MALDI matrix, 2,5-dihydroxybenzoic acid (DHB) were purchased from
Sigma-Aldrich (Milwaukee, WI, USA). Chloroform, methyl iodide, dimethylsulfoxide (DMSO), sodium acetate $(\mathrm{NaOAc})$, hydrochloric acid $(\mathrm{HCl})$, and sodium chloride $(\mathrm{NaCl})$ were received from EM Science (Gibbstown, NJ, USA). Silica-based strong anion-exchange spin-columns were purchased from ZirChrom Separations, Inc. (Anoka, MN, USA).

\section{Release of N-Glycans from Bovine Thyroid-Stimulating Hormone (bTSH)}

N-Glycans were liberated from bTSH glycoprotein through the use of PNGase F, according to our previously reported procedure [35]. Briefly, $100 \mu \mathrm{g}$ (2 nmol) of the glycoprotein was dissolved in $50 \mu \mathrm{L} 100 \mathrm{mM} \mathrm{pH}$ 7.5 phosphate buffer containing $0.5 \%$ SDS. The mixture was then heated at $95^{\circ} \mathrm{C}$ in a water bath for $10 \mathrm{~min}$. Next, NP-40 was added to neutralize the negative charges of SDS before the addition of PNGase F (0.5 $\mathrm{mU})$. The sample was then incubated overnight at $37^{\circ} \mathrm{C}$. PNGase $\mathrm{F}$ was added again $4 \mathrm{~h}$ before the termination of the $37^{\circ} \mathrm{C}$ incubation. Finally, samples were vacuum-dried before permethylation.

\section{Solid-Phase Permethylation}

Solid-phase permethylation and deuteromethylation were performed according to our previously published procedure- $[30,31,36]$. Briefly, $\mathrm{NaOH}$ beads were packed in a spin-column to about $3 \mathrm{~cm}$ depth using acetonitrile. The spin-column was then centrifuged and washed several times with $50-\mu \mathrm{L}$ aliquots of DMSO. Vacuum-dried sulfated oligosaccharide standard and $\mathrm{N}$-glycans derived from bTSH were dissolved in $90 \mu \mathrm{L}$ DMSO and $40 \mu \mathrm{L}$ methyl iodide (or deuteromethyl iodide). A $1-\mu \mathrm{L}$ aliquot of water was then added to prevent peeling reactions [37]. The sample solution was then centrifuged at low speed. Samples were recycled through the spin-column eight times. The spin-column was finally washed with an additional $50-\mu \mathrm{L}$ aliquot of DMSO. Glycans released from $\sim 100 \mu \mathrm{g}(2 \mathrm{nmol}) \mathrm{bTSH}$ glycoprotein were used in this step.

\section{$C_{18}$ Extraction}

A 2-mL aliquot of $0.1 \%$ trifluoroacetic acid (TFA) aqueous solution was added to permethylated samples before a solid-phase extraction using preconditioned $\mathrm{C}_{18}$ cartridges. TFA was used here as an ion-pairing agent. All $\mathrm{C}_{18}$ cartridges were preconditioned with a 2-mL ethanol aliquot and a 2-mL water aliquot. After applying samples, cartridges were washed with a 2-mL water aliquot, while permethylated sugars were eluted with a 1.8-mL aliquot of 90:5:5 ACN:water:isopropanol solution. The eluent was vacuum-dried. 


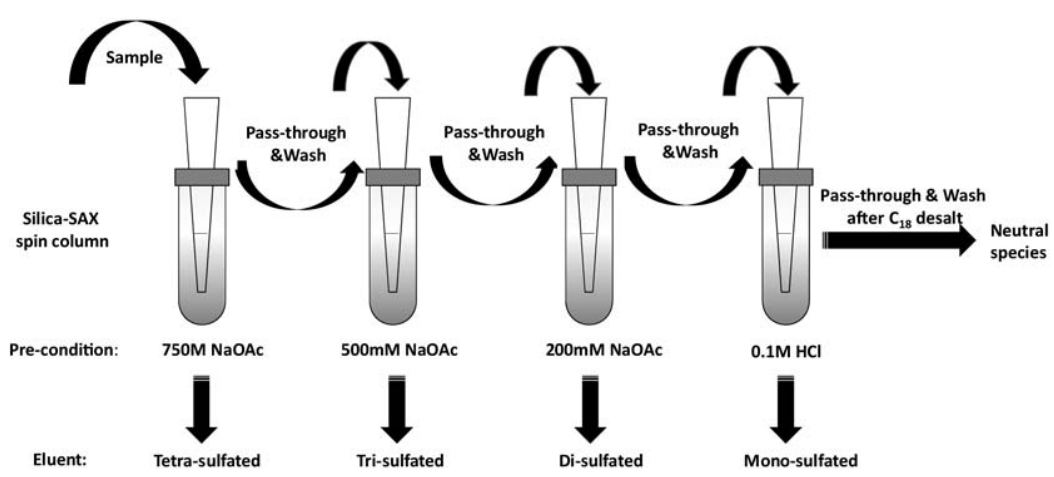

Figure 1. Experimental procedure for sequential enrichment. Approximately $10 \mathrm{mg}$ SAX material was packed in each spin-column.

\section{Sequential Enrichment Using Silica-based Strong Anion-exchange (SAX) Spin-Column}

A series of SAX spin-columns were preconditioned using double-bed volumes of 750, 500, 200, 100, $50 \mathrm{mM}$ $\mathrm{NaOAc}$ and $0.1 \mathrm{M} \mathrm{HCl}$ aqueous solution. The permethylated sample was suspended in 5\% ACN aqueous solution. Next, the sample containing either permethylated standard glycan structures or permethylated Nglycans derived from bTSH was sequentially passed through each preconditioned SAX spin-column following high to low NaOAc concentration. The sample was passed through spin-columns three times to ensure adequate interaction. Next, 20-fold bed-volume of water was used to wash off the nonspecifically bound components. Finally, the column-bound sample components were eluted using 5\% ACN aqueous solution containing $1 \mathrm{M} \mathrm{NaCl}$. The fractions were then desalted using a $\mathrm{C}_{18}$ column and vacuum-dried before MS analysis. A mixture composed of 200 pmol of each sulfated oligosaccharide standard was used for optimization, while $100 \mu \mathrm{g}$ (2 nmol) bTSH glycoprotein was used to demonstrate the ability to enrich sulfated glycan structures derived from a biologically relevant glycoprotein.

\section{MALDI Mass Spectrometry}

Mass-spectrometric analysis was performed on an Applied Biosystems 4800 Proteomics Analyzer (Applied Biosystems, Framingham, MA, USA). The Nd:YAG laser of this instrument has a wavelength of $355 \mathrm{~nm}$. For the MS analysis, the positive-ion mode with a reflectron was used. MS data were further processed using DataExplorer 4.6 (Applied Biosystems). Positive MALDI-CID spectra were obtained using $1 \mathrm{kV}$ collision energy defined by the potential difference between the source and the collision cell. Air was used as the collision gas at a pressure of $8.0 \times \mathrm{E}^{-6}$ torr to enhance cross-ring fragmentation.

The vacuum-dried sample was resuspended in a 1:1 methanol:water mixture and mixed with the MALDI matrix at 1:1 ratio. The MALDI matrix consisting of 10 $\mathrm{mg} / \mathrm{mL}$ DHB was prepared by dissolving DHB in 1:1 methanol:2 mM sodium acetate aqueous solution.

\section{Results and Discussion}

\section{Method Optimization Using Sulfated Oligosaccharide Standards}

As the binding of sulfated glycans on the SAX material is determined by a charge state of the sample along with the density of positively charged quaternary amine groups (active sites) on the stationary-phase surface, $\mathrm{NaOAc}$ solution is used here to adjust the active-site density on the SAX column by neutralizing a population of the positively charged quaternary amine groups with $\mathrm{OAc}^{-}$. Accordingly, the percentage of neutralized active sites can be tuned by the amount of $\mathrm{NaOAc}$ applied. As the result, the binding ability of SAX material can be adjusted to the extent that the molecules with the highest sulfation state present in the sample are reversibly retained. For example, the active-site density on the SAX material after $750 \mathrm{mM} \mathrm{NaOAc}$ condition is lowered to the extent that the tetrasulfated species are retained; similarly, $500 \mathrm{mM} \mathrm{NaOAc-conditioned}$ SAX column retains trisulfated glycans. Therefore, by applying the sample sequentially through a series of NaOAc-conditioned SAX columns, glycans are expected to be sequentially separated according to their sulfation states as mentioned above.

We first studied the ability of SAX material to bind permethylated sulfated oligosaccharide standards at different sodium acetate $(\mathrm{NaOAc})$ concentrations. A mixture of permethylated mono-, di-, tri-, and tetrasulfated oligosaccharide standards was sequentially passed through a series of SAX spin-columns, which were preconditioned with 200,500, and $750 \mathrm{NaOAc}$ aqueous solution and $0.1 \mathrm{M} \mathrm{HCl}$ (Figure 1). As depicted in Figure 2a, the MALDI-MS signals of the highly sulfated oligosaccharides were suppressed by those of the less sulfated counterparts. Although the mixture was composed of equal amounts of all species, monosulfated oligosaccharides appeared to exhibit dominant signals (Figure 2b). Di- and trisulfated oligosaccharides were detected at significantly lower intensities, while tetrasulfated oligosaccharides were not detected due to a suppressed ionization. Upon passing the sample through SAX spin-columns as described above, sulfated 


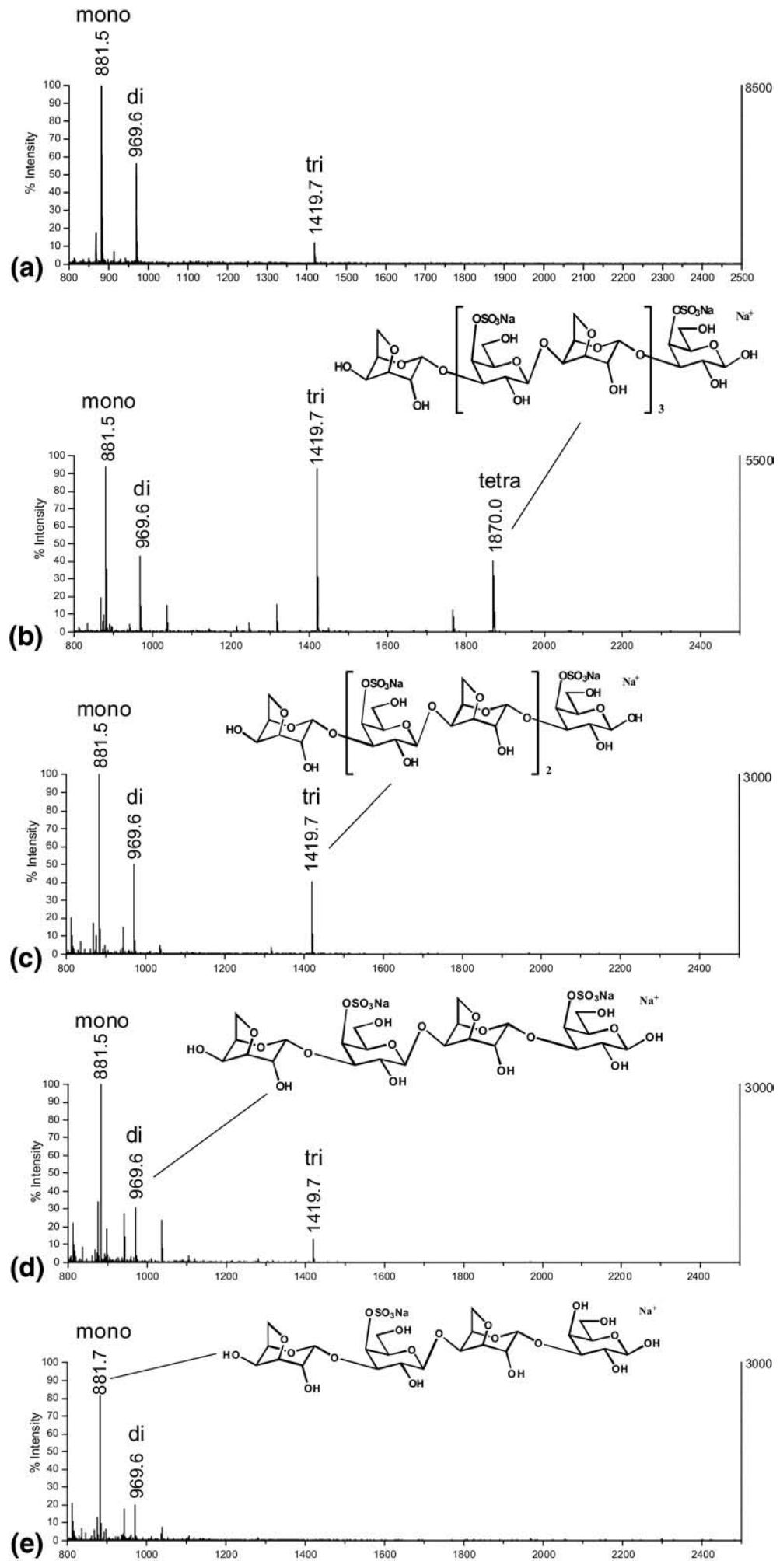

Figure 2. MALDI-MS of a permethylated standard sulfated oligosaccharides mixture before (a) and after sequential separation. The mixture is composed of mono-, di-, tri-, and tetrasulfated oligosaccharides. It was sequentially passed through a series of silica-based strong anion-exchange column, which had been preconditioned with $750 \mathrm{mM} \mathrm{NaOAc}$ (b), $500 \mathrm{mM} \mathrm{NaOAc}$ (c), $200 \mathrm{mM} \mathrm{NaOAc}$ (d), and $0.1 \mathrm{M} \mathrm{HCl}(\mathbf{e})$. Elution was attained through the use of $1 \mathrm{M} \mathrm{NaCl}$ with $5 \% \mathrm{ACN}$ and was desalted by $\mathrm{C}_{18}$ spin-column. 
oligosaccharides were partially resolved according to the number of sulfate groups associated with each oligosaccharide, as shown in Figure 2b-e. Tetrasulfated oligosaccharides were observed exclusively in the eluent of the SAX spin-column preconditioned with 750 $\mathrm{mM} \mathrm{NaOAc}$, while trisulfated species were enriched with respect to the mono- and disulfated standards under this condition (Figure 2b). Mainly trisulfated and part of disulfated species were enriched in the eluent of the SAX spin-column preconditioned with $500 \mathrm{mM}$ $\mathrm{NaOAc}$, while tetrasulfated species were not detected (Figure 2c). The MALDI mass spectrum of the eluent of the SAX spin-column preconditioned with $200 \mathrm{mM}$ $\mathrm{NaOAc}$ (Figure 2d) consisted of the signals corresponding to the mono-, di-, and trisulfated oligosaccharides. However, the signals of both di- and trisulfated oligosaccharides were weaker. Finally, under $0.1 \mathrm{M} \mathrm{HCl}$ condition (Figure 2e), both mono- and disulfated oligosaccharides were observed; however, monosulfated oligosaccharides appeared to be more dominant. Monoand disulfated oligosaccharides were observed in all eluents because there is always sufficient active site density on an SAX column to prompt adequate interaction for retaining these sulfated structures. On the other hand, the interaction can also be compromised by $1 \mathrm{M}$ $\mathrm{NaCl}$ elution condition. The efficiency of the described sequential separation cannot be solely determined on the basis of their relative intensities for the different sulfated oligosaccharides, since the ionization efficiency decreases as the sulfation state increases. Nevertheless, the optimized condition for each sulfate state can be empirically determined by comparing the relative intensities.

\section{Enrichment of Sulfated N-Glycans Derived from Bovine Thyroid-Stimulating Hormone}

Next, we applied the above described procedure to the enrichment of glycans derived from bovine thyroidstimulating hormone (bTSH). This glycoprotein features mono- and disulfated glycan structures (Figure 3a) $[2,33$, 34]. N-glycans from bTSH were released, permethylated, and sequentially enriched according to the optimized procedure described above. Each spectrum was acquired using $\sim 5 \mu \mathrm{g}$ (100 pmol) of bTSH glycoprotein.

Sulfated structures observed for each condition as well as their relative intensities before elution and in the different eluted fractions of the different SAX spincolumns are summarized in Table 1. No glycan was observed in the eluent of the $500 \mathrm{mM} \mathrm{NaOAc}$ preconditioned SAX spin-column (Figure 3b), thus confirming the absence of glycan structures possessing more than two sulfate groups. All disulfated glycans were retained on the $200 \mathrm{mM} \mathrm{NaOAc}$ preconditioned SAX spincolumn (Figure 3c), while their intensities were significantly enhanced compared with those from the same sample analyzed without the described sequential en- richment (Figure 3a). Again, this confirms that this condition is optimized for selectively retaining the disulfated glycans. Similar to the sulfated oligosaccharide standards, monosulfated glycan structures were observed in all eluents. Interestingly, a previously undetected disulfated structure $(\mathrm{m} / \mathrm{z} 2327.7)$ was observed after the enrichment (Figure 3c, Table 1). It might be that without fractionation, this structure was suppressed by the other structures because of its low abundance and its degree of sulfation. This component was selectively enriched relative to the monosulfated glycans as a result of the sequential enrichment procedure, thus prompting an effective ionization/detection.

The eluents of $0.1 \mathrm{M} \mathrm{HCl}$ preconditioned SAX spincolumn did not include any disulfated structures, confirming that the isolation of disulfated glycans has been efficient. Three previously undetected monosulfated glycans $(\mathrm{m} / \mathrm{z} 2331.8,2413.9$, and $2588.2 \mathrm{Da})$ were observed in the eluent of the $0.1 \mathrm{M} \mathrm{HCl}$-preconditioned SAX spin-column, suggesting a selective enrichment of monosulfated species under this condition. Moreover, two important features of this method can be deduced from this observation. First, the sample components with different sulfation states can be selectively and sequentially enriched. Second, the selectivity is relatively high even in the case of low-abundance species. On the other hand, the relative intensities of monosulfated glycans in the eluent of the $0.1 \mathrm{M} \mathrm{HCl}$-preconditioned SAX spin-column (Figure 3d, Table 1) remained the same compared with those without sequential enrichment. It indicates that the sulfation state dictates the separation during the procedure without any structural discrimination. It also suggests that the role of ion-exchange principle as the dominant separation mechanism of SAX material is unchanged after NaOAc preconditioning. Therefore, an adaptation of this method to an automated system such as HPLC is plausible without major modifications.

To further demonstrate the advantage of selective enrichment, we performed collision-induced dissociation (CID) MS/MS analysis on a disulfated glycan $(\mathrm{m} / \mathrm{z}$ $2051.7 \mathrm{Da}$ ) before (Figure 4a) and after (Figure 4b) sequential enrichment. These spectra were recorded from $5 \mu \mathrm{g}$ glycoprotein eluted from the $200 \mathrm{mM}$ NaOAc-preconditioned SAX spin-column. The overall signal-to-noise ratio $(\mathrm{S} / \mathrm{N})$ and the number of observed fragments were enhanced after the enrichment. More specifically, only two fragment ions were observed for the sample before treatment, whereas five fragment ions were recorded after the treatment (Figure 4). Therefore, sulfated glycans can be better characterized by means of sequential enrichment.

To examine the specificity of the described approach towards sulfated glycans, we applied this method to a mixture of glycans released from ribonuclease $B$, fetuin, $\alpha_{1}$-acid glycoprotein, and bTSH (mass ratio, 1:5:10:20). Such a mixture contains high-mannose glycans originating from ribonuclease $B$, sialylated glycan structures originating from fetuin, and fucosylated and sialylated 

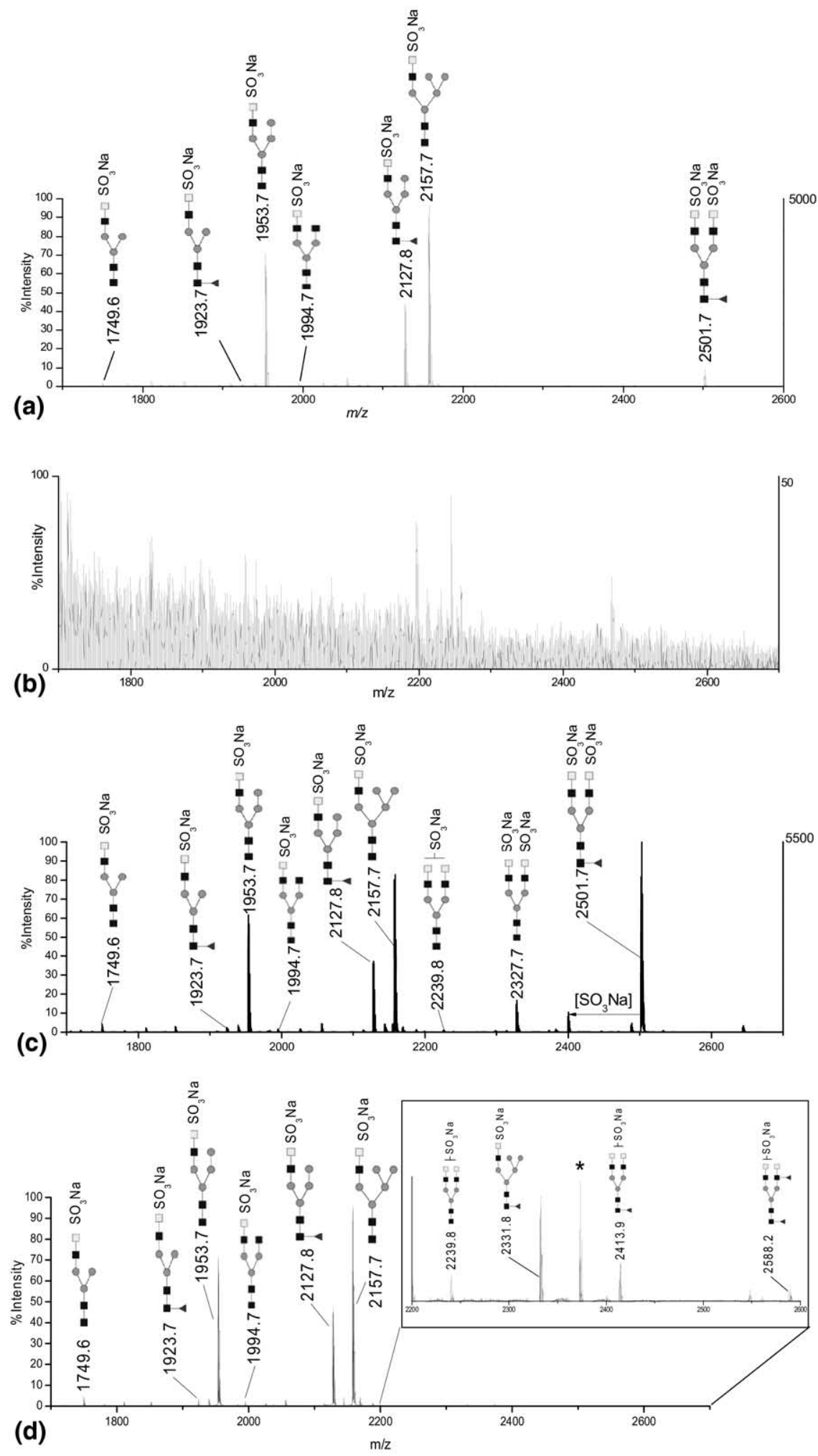

Figure 3. MALDI-MS of permethylated N-glycans derived from $5 \mu \mathrm{g}$ bTSH glycoprotein before (a) and after sequential enrichment. Permethylated glycan from bTSH was passed through $500 \mathrm{mM}$ $\mathrm{NaOAc}(\mathbf{b}), 200 \mathrm{mM} \mathrm{NaOAc}(\mathbf{c})$, and $0.1 \mathrm{M} \mathrm{HCl}(\mathbf{d})$, preconditioned silica-based strong anion-exchange spin-column sequentially. Disulfated glycans were enriched in $200 \mathrm{mM} \mathrm{NaOAc}$ condition. Structures of the identified sulfated glycans were labeled on the spectrum. The peak marked with asterisk corresponds to unidentified structure. 
Table 1. Sulfated glycan structures observed before and after sequential enrichment for each condition. All the sulfate groups are located at the 4-position of the GalNAc residues ${ }^{2,33}$ for each structure shown

\begin{tabular}{|c|c|c|c|c|c|c|}
\hline \multirow[b]{3}{*}{ Structure } & \multirow{2}{*}{\multicolumn{2}{|c|}{$m / z(\mathrm{Da})^{\mathrm{a}}$}} & \multicolumn{4}{|c|}{ Relative intensities $^{\text {b }}$} \\
\hline & & & \multirow[b]{2}{*}{ Before } & \multicolumn{3}{|c|}{ After } \\
\hline & Calc'd & Observed & & $500 \mathrm{mM} \mathrm{NaOAc}$ & $200 \mathrm{mM} \mathrm{NaOAc}$ & $0.1 \mathrm{M} \mathrm{HCl}$ \\
\hline & 1749.8 & 1749.6 & 0.8 & $\mathrm{~N} / \mathrm{D}$ & 0.7 & 1.3 \\
\hline & 1923.8 & 1923.7 & 0.4 & $\mathrm{~N} / \mathrm{D}$ & 0.3 & 0.4 \\
\hline & 1953.9 & 1953.7 & 33.5 & $\mathrm{~N} / \mathrm{D}$ & 23.0 & 33.8 \\
\hline & 1994.9 & 1994.7 & 0.4 & $\mathrm{~N} / \mathrm{D}$ & 0.2 & 0.4 \\
\hline & 2127.9 & 2127.8 & 21 & $N / D$ & 9.8 & 21.1 \\
\hline a & 2157.9 & 2157.7 & 41.8 & $\mathrm{~N} / \mathrm{D}$ & 26.3 & 42.2 \\
\hline 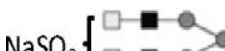 & 2240.0 & 2239.8 & $N / D$ & $N / D$ & 0.2 & 0.2 \\
\hline a & 2332.0 & 2331.8 & $N / D$ & $N / D$ & $N / D$ & 0.2 \\
\hline & 2414.1 & 2413.9 & $N / D$ & $\mathrm{~N} / \mathrm{D}$ & N/D & 0.2 \\
\hline $\bar{\nabla}$ & 2588.2 & 2588.2 & $N / D$ & $\mathrm{~N} / \mathrm{D}$ & $\mathrm{N} / \mathrm{D}$ & 0.2 \\
\hline $\begin{array}{l}\mathrm{NaSO}_{3}=- \\
\mathrm{NaSO}_{3}=-\end{array}$ & 2327.9 & 2327.7 & $N / D$ & $N / D$ & 6.6 & $N / D$ \\
\hline $\begin{array}{l}\mathrm{NaSO}_{3} \square=- \\
\mathrm{NaSO}_{3} \square=-\end{array}$ & 2501.9 & 2501.7 & 2.1 & $N / D$ & 32.9 & $\mathrm{~N} / \mathrm{D}$ \\
\hline
\end{tabular}

$\mathrm{N} / \mathrm{D}=$ not detected

$\square$ GalNAc, $\square$ GIcNAc, $\bigcirc$ Mannose, $\Delta$ Fucose.

${ }^{a}$ Monoisotopic $\mathrm{m} / \mathrm{z}$ of $[\mathrm{M}+\mathrm{Na}]^{+}$measured for permethylated glycans from bTSH. All sulfates were sodiated.

${ }^{\mathrm{b}}$ Relative intensities were calculated with respect to the sum of intensities for observed structures.

glycan structures originating from $\alpha_{1}$-acid glycoprotein. This mixture is a realistic representative of glycan structures present in a biological system. The mass ratio of each glycoprotein in the mixture was optimized to obtain comparable signals from each structure. The permethylated mixture was sequentially passed through $200 \mathrm{mM} \mathrm{NaOAc}$ and $0.1 \mathrm{M} \mathrm{HCl}$ preconditioned SAX spin-columns and desalted as described above. The MALDI mass spectra of the mixture before and after sequential enrichments are depicted in Figure 5. Before the sequential enrichment (Figure 5a), only five of the sulfated glycan structures originating from bTSH (boxed $\mathrm{m} / \mathrm{z}$ ) were observable, while the glycans from the other three glycoproteins appeared in high abundance. This spectrum clearly demonstrates MALDI suppression of sulfated glycans in the presence of neutral structures. The structures that were not retained on the SAX spin-columns were recovered from pass-through and wash solutions (Figure 5b). All the neutral structures and the sialylated glycans presented in the mixture were observed in this fraction, indicting a selective enrichment power of this approach for sulfated glycans. The sialylated glycans were not retained on the SAX column due to the previous permethylation, which eliminates the negative charge commonly associated with such structures. 

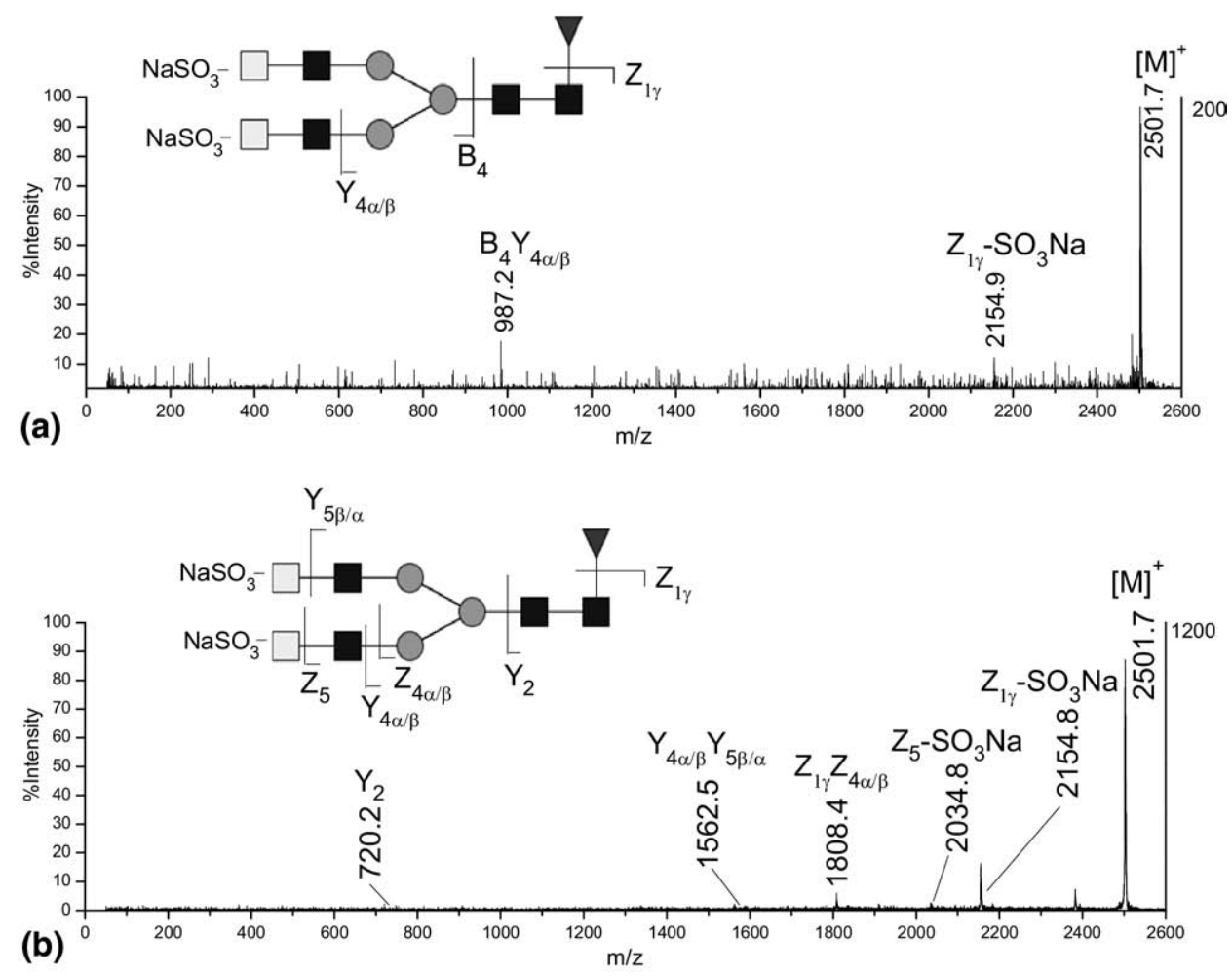

Figure 4. CID MS/MS of a disulfated structure $(m / z=2501.7 \mathrm{Da})$ before (a) and after (b) sequential enrichment (200 mM NaOAc preconditioned). Each spectrum was recorded from glycans obtained from $3 \mu \mathrm{g}$ glycoprotein, while glycan ions were sodiated.

On the other hand, the MALDI-MS signals of all sulfated glycans originating from bTSH were significantly enhanced after the sequential enrichment (Figure $5 c, d)$. All of the sulfated glycans were observed in the eluent while none of the neutral glycans were retained on the $200 \mathrm{mM}$ NaOAc-preconditioned SAX spin-column. Some of the sialylated glycans $(\mathrm{m} / \mathrm{z}$ 3602.6) were observed, but at a very low intensity. We believe this is due to a limited secondary interaction, which does not substantially affect the efficiency of the described enrichment protocol. Disulfated structures $(\mathrm{m} / \mathrm{z}$ 2327.8, 2501.8) were enriched in $200 \mathrm{mM} \mathrm{NaOAc}$-preconditioned SAX spin-column (Figure 5c), but were not observed in the eluent of the $0.1 \mathrm{M} \mathrm{HCl}$-preconditioned SAX spincolumn (Figure 5d), thus confirming the high selectivity of our enrichment approach.

\section{Conclusions}

Sequential enrichment using a SAX spin-column system allows separating glycans according to the number of sulfate groups associated with such structures, resulting in a substantial increase in their MALDI-MS signals. The binding capacities of the silica-based SAX materials can be modulated by the use of different concentrations of $\mathrm{NaOAc}$, thus fractionating sulfated glycans according to their different numbers of sulfate groups. The approach is particularly effective when the samples are initially permethylated to neutralize the sialylated species, as well as to enhance MALDI-MS ionization of sulfated glycans. The described approach requires minimum sample amounts ( $\mu \mathrm{g}$ scale) and can be accomplished within $20 \mathrm{~min}$. The procedure was effective in

Figure 5. MALDI-MS spectra of permethylated N-glycan derived from a 1:5:10:20 mixture of ribonuclease $\mathrm{B}$, fetuin, $\alpha_{1}$-acid glycoprotein, and bovine thyroid-stimulating hormone before (a), after (b), (c), (d) sequential enrichment. The mass ratio of glycoproteins used was optimized to observe comparable signal from each component. N-glycan structures derived from ribonuclease B, fetuin, and $\alpha_{1}$-acid glycoprotein were labeled in (a), while the glycans from bTSH were squared. (b) Shows the MALDI-MS spectra of not-retained species that were recovered from the pass-through and wash. Mixture of permethylated glycans was passed sequentially through $200 \mathrm{mM} \mathrm{NaOAc}$ (c) and $0.1 \mathrm{M} \mathrm{HCl}$ (d) preconditioned silica-based strong anion-exchange spin-column. Disulfated glycans were enriched under the $200 \mathrm{mM} \mathrm{NaOAc}$ condition. Structures of identified sulfated glycans were labeled on the spectrum. The peak marked with asterisk corresponds to an unidentified structure. Key to symbols: [open square] GalNAc, [filled square] GlcNAc, [filled circle] mannose, [filled triangle] fucose, [filled diamond] sialic acid, [open circle] galactose. 

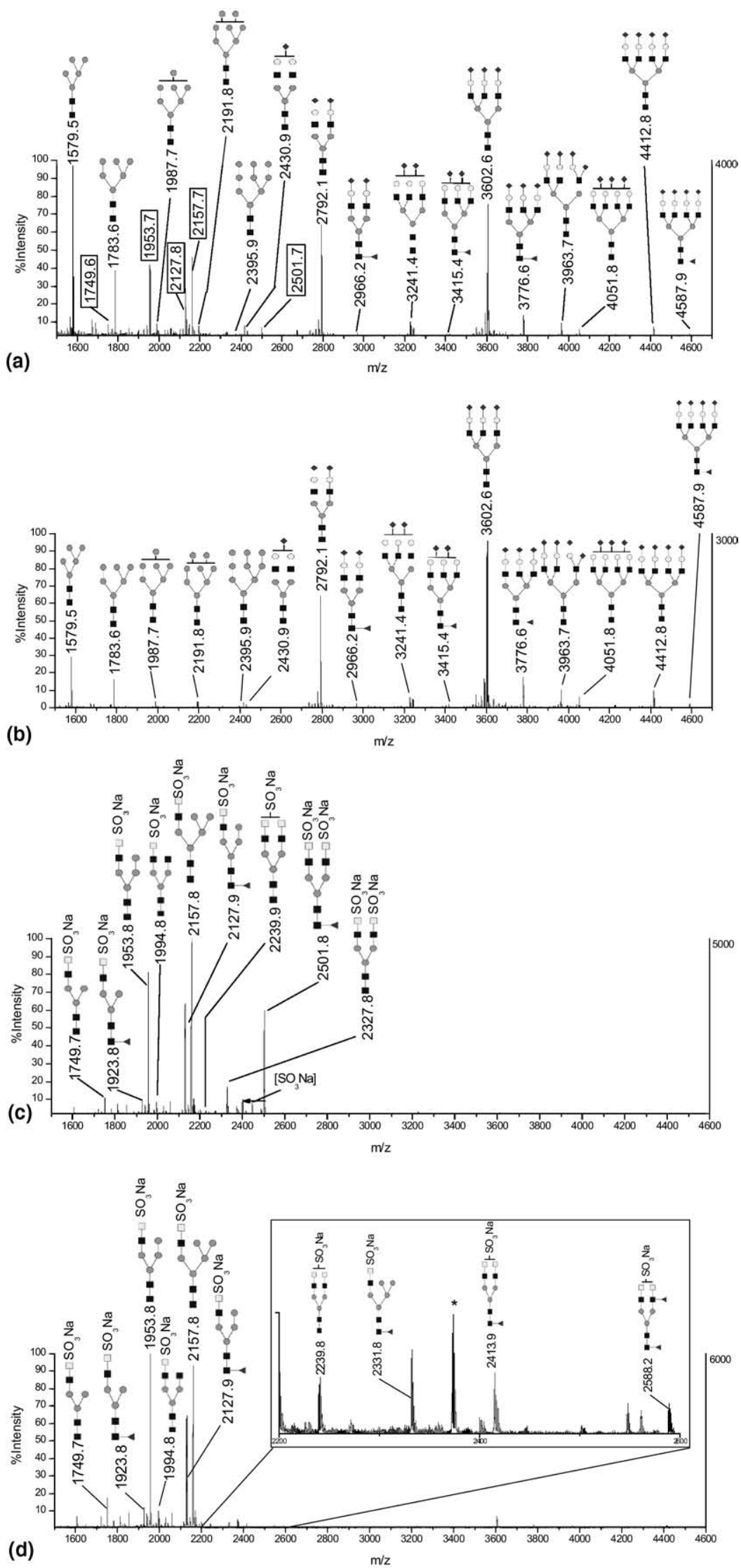
enhancing the signals of highly sulfated structures. It also allowed the isolation of sulfated structures from complex glycan mixture containing permethylated neutral and sialylated glycans. Moreover, enhancing the MALDI-MS signals allowed a more informative tandem MS analysis. This approach appears quite effective for MALDI-MS and MS/MS characterization of sulfated structures originating from various biological samples.

\section{Acknowledgments}

The authors acknowledge primary support for this work by grant no. GM24349 from the National Institute of General Medical Sciences, U.S. Department of Health and Human Services. Additional support was provided by NIH/NCRR for the National Center for Glycomics and Glycoproteomics (NCGG) at Indiana University (grant no. RR018942).

\section{References}

1. Baenziger, J. U.; Kuma, S.; Brodbeck, R. M.; Smith, P. L.; Beranek, M. C. Circulatory Half-Life but not Interaction with the Lutropin/Chorionic Gonadotropin Receptor is Modulated by Sulfation of Bovine Lutropin Oligosaccharides. Proc. Nat. Acad. Sci. U.S.A. 1992, 89(1), 334-338.

2. Baenziger, J. U.; Green, E. D. Pituitary Glycoprotein Hormone Oligosaccharides: Structure, Synthesis, and Function of the AsparagineLinked Oligosaccharides on Lutropin, Follitropin, and Thyrotropin. Biochim. Biophys. Acta Rev. Biomembr. 1988, 947(2), 287-306.

3. Stockell Hartree, A.; Renwick, A. G. Molecular Structures of Glycoprotein Hormones and Functions of Their Carbohydrate Components. Biochem. J. 1992, 287(3), 665-679.

4. Imai, Y.; Lasky, L. A.; Rosen, S. D. Sulphation Requirement for GlyCAM-1, an Endothelial Ligand for L-Selectin. Nature 1993, 361(6412), 555-557.

5. Imai, Y.; Singer, M. S.; Fennie, C.; Lasky, L. A.; Rosen, S. D. Identification of a Carbohydrate-Based Endothelial Ligand for a Lymphocyte Homing Receptor. J. Cell Biol. 1991, 113(5), 1213-1221.

6. Hemmerich, S.; Bertozzi, C. R.; Leffler, H.; Rosen, S. D. Identification of the Sulfated Monosaccharides of GlyCAM-1, an Endothelial-Derived Ligand for L-Selectin. Biochemistry 1994, 33(16), 4820-4829.

7. Shilatifard, A.; Merkle, R. K.; Helland, D. E.; Welles, J. L.; Haseltine, W. A.; Cummings, R. D. Complex-Type N-Linked Oligosaccharides of gp120 from Human Immunodeficiency Virus Type 1 Contain Sulfated N-Acetylglucosamine. J. Virol. 1993, 67(2), 943-952.

8. Fukuda, M.; Hiraoka, N.; Akama, T. O.; Fukud, M. N. Carbohydratemodifying Sulfotransferases: Structure, Function, and Pathophysiology. J. Biol. Chem. 2001, 276.

9. Martini, R.; Xin, Y.; Schmitz, B.; Schachner, M. The L2/HNK-1 Carbohydrate Epitope Is Involved in the Preferential Outgrowth of Motor Neurons on Ventral Roots and Motor Nerves. Eur. J. Neurosci. 1992, 4(7), 628-639.

10. Chance, D. L.; Mawhinney, T. P. Disulfated Oligosaccharides Derived from Tracheobronchial Mucous Glycoproteins of a Patient Suffering from Cystic Fibrosis. Carbohydr. Res. 1996, 295, 157-177.

11. Fuster, M. M.; Esko, J. D. The Sweet and Sour of Cancer: Glycans as Novel Therapeutic Targets. Nat. Rev. Cancer 2005, 5(7), 526-542.

12. Xiong, L.; Andrews, D.; Regnier, F. Comparative Proteomics of Glycoproteins Based on Lectin Selection and Isotope Coding. J. Proteome Res. 2003, 2(6), 618-625.

13. Thomsson, K. A.; Karlsson, N. G.; Hansson, G. C. Liquid ChromatographyElectrospray Mass Spectrometry as a Tool for the Analysis of Sulfated Oligosaccharides from Mucin Glycoproteins. J. Chromatogr. A 1999, 854(1/2), 131-139.

14. Ohtsubo, K.; Marth, J. D. Glycosylation in Cellular Mechanisms of Health and Disease. Cell 2006, 125, 855-867.
15. Freeze, H.; Wolgast, D. Structural Analysis of N-Linked Oligosaccharides from Glycoproteins Secreted by Dictyostelium discoideum. Identification of Mannose 6-sulfate. J. Biol. Chem. 1986, 261(1), 127-134.

16. Bowman, K. G.; Cook, B. N.; de Graffenried, C. L.; Bertozzi, C. R Biosynthesis of L-Selectin Ligands: Sulfation of Sialyl Lewis x-Related Oligosaccharides by a Family of GlcNAc-6-Sulfotransferases. Biochemistry 2001, 40(18), 5382-5391.

17. Hard, K.; Van Zadelhoff, G.; Moonen, P.; Kamerling, J. P.; Vliegenthart, J. F. G. The Asn-Linked Carbohydrate Chains of Human Tamm-Horsfall Glycoprotein of One Male. Eur. J. Biochem. 1992, 209, 895-915.

18. van Rooijen, J. J. M.; Kamerling, J. P.; Vliegenthart, J. F. G. Sulfated Di-, Tri-, and Tetra-Antennary N-Glycans in Human Tamm-Horsfall Glycoprotein. Eur. J. Biochem. 1998, 256(2), 471-487.

19. Zaia, J. Mass Spectrometry of Oligosaccharides. Mass Spectrom. Rev. 2004, 23, 161-227.

20. Mechref, Y.; Novotny, M. V. Miniaturized Separation. Techniques in Glycomic Investigations. J. Chromatogr. B 2006, 841, 65-78.

21. Mechref, Y.; Novotny, M. V. Structural Investigations of Glycoconjugates at High Sensitivity. Chem. Rev. 2002, 102, 321-370.

22. Jiang, H.; Irungu, J.; Desaire, H. Enhanced Detection of Sulfated Glycosylation Sites in Glycoproteins. J. Am. Soc. Mass Spectrom. 2005, 16(3), 340-348.

23. Irungu, J.; Dalpathado, D. S.; Go, E. P.; Jiang, H.; Ha, H.-V.; Bousfield, G. R.; Desaire, H. Method for Characterizing Sulfated Glycoproteins in a Glycosylation Site-Specific Fashion, Using Ion Pairing and Tandem Mass Spectrometry. Anal. Chem. 2006, 78(4), 1181-1190.

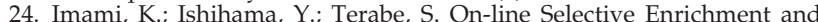
Ion-pair Reaction for Structural Determination of Sulfated Glycopeptides by Capillary Electrophoresis-Mass Spectrometry. J. Chromatogr. A 2008, 1194(2), 237-242.

25. Barboza, M.; Duschak, V. G.; Fukuyama, Y.; Nonami, H.; Erra-Balsells, R.; Cazzulo, J. J.; Couto, A. S. Structural Analysis of the N-glycans of the Major Cysteine Proteinase of Trypanosoma cruzi. FEBS J. 2005, 272, 3803-3815.

26. Murakami, T.; Natsuka, S.; Nakakita, S.-I.; Hase, S. Structure Determination of a Sulfated N-Glycans, Candidate for a Precursor of the Selectin Ligand in Bovine Lung. Glycoconj. J. 2007, 24(4), 195-206.

27. Lo-Guidice, J.-M.; Herz, H.; Lamblin, G.; Plancke, Y.; Roussel, P.; Lhermitte, M. Structures of Sulfated Oligosaccharides Isolated from the Respiratory Mucins of a Nonsecretor $(\mathrm{O}$, Lea $+\mathrm{b}-)$ Patient Suffering from Chronic Bronchitis. Glycoconj. J. 1997, 14(1), 113-125.

28. Yagi, H.; Takahashi, N.; Yamaguchi, Y.; Kimura, N. Uchimura, K Kannagi, R.; Kato, K. Development of Structural Analysis of Sulfated N-Glycans by Multidimensional High Performance Liquid Chromatography Mapping Methods. Glycobiol. 2005, 15, 1051-1060.

29. Hermentin, P.; Witzel, R.; Doenges, R.; Bauer, R.; Haupt, H.; Patel, T. Parekh, R. B.; Brazel, D. The Mapping by High-pH Anion-Exchange Chromatography with Pulsed Amperometric Detection and Capillary Electrophoresis of the Carbohydrate Moieties of Human Plasma $[\alpha] 1-$ acid Glycoprotein. Anal. Biochem. 1992, 206(2), 419-429.

30. Kang, P.; Mechref, Y.; Novotny, M. V. High-Throughput Solid-phase Permethylation of Glycans Prior to Mass Spectrometry. Rapid Commun. Mass Spectrom. 2008, 22(5), 721-734.

31. Kang, P.; Mechref, Y.; Klouckova, I.; Novotny, M. V. Solid-Phase Permethylation of Glycans for Mass Spectrometric Analysis. Rapid Commun. Mass Spectrom. 2005, 19, 3421-3428.

32. Kopaciewicz, W.; Regnier, F. E. Mobile Phase Selection for the HighPerformance Ion-Exchange Chromatography of Proteins. Anal. Biochem. 1983, 133(1), 251-259.

33. Wheeler, S. F.; Harvey, D. J. Extension of the In-Gel Release Method for Structural Analysis of Neutral and Sialylated N-Linked Glycans to the Analysis of Sulfated Glycans: Application to the Glycans from Bovine Thyroid-Stimulating Hormone. Anal. Biochem. 2001, 296(1), 92-100.

34. Morelle, W.; Donadio, S.; Ronin, C.; Michalski, J.-C. Characterization of N-Glycans of Recombinant Human Thyrotropin using Mass Spectrometry. Rapid Commun. Mass Spectrom. 2006, 20(3), 331-345.

35. Mechref, Y.; Novotny, M. V. Mass Spectrometric Mapping and Sequencing of N-Linked Oligosaccharides Derived from Submicrogram Amounts of Glycoproteins. Anal. Chem. 1998, 70(3), 455-463.

36. Devakumar, A.; Mechref, Y.; Kang, P.; Novotny, M. V.; Reilly, J. P. Laser-Induced Photofragmentation of Neutral and Acidic Glycans Inside an Ion-Trap Mass Spectrometer. Rapid Commun. Mass Spectrom. 2007, 21(8), 1452-1460.

37. Ciucanu, I.; Costello, C. E. Elimination of Oxidative Degradation during the Per-O-Methylation of Carbohydrates. J. Am. Chem. Soc. 2003, 125, 16213-16219. 